\title{
Metabolic Syndrome: a challenging health Issue in highly urbanized Union Territory of north India
}

\author{
Chetna Mangat ${ }^{1 *}$, NK Goel ${ }^{1}$, Dinesh K Walia', Neeraj Agarwal ${ }^{1}$, Munesh K Sharma', Jasbinder Kaur ${ }^{2}$, Ram Singh ${ }^{3}$, \\ Gagandeep Singh ${ }^{4}$
}

\begin{abstract}
Objectives: 1. To determine the prevalence of Metabolic Syndrome in adults aged 18 years and above in Chandigarh, India. 2. To determine the socio-demographic factors associated with MS. 3. To determine the agreement between IDF (International Diabetes federation definition) and ATP-III (National Cholesterol Education Program Expert Panel on Detection, Evaluation, and Treatment of High Blood Cholesterol in Adults criteria).

Methods: In a community based cross-sectional study, total 605 subjects aged 18 yrs and above were studied using multistage random sampling.

Results: Prevalence of Metabolic Syndrome was estimated by using IDF and ATP-III criteria. By IDF, Metabolic Syndrome was found in 287 (47.4\%) subjects and it was more prevalent among females 171 (59.6\%) as compared to males 116 (40.4\%). By applying ATP-III overall prevalence was less i.e. 233 (38.5\%) but again its prevalence was more among females 141 (44.8\%) than males 116 (39.5\%). Higher socioeconomic status, sedentary occupation and high body mass index were significantly associated with Metabolic Syndrome.
\end{abstract}

Conclusions: Metabolic Syndrome is a major health problem in the region and proper emphasis should be given on its prevention and control.

\section{Introduction}

MS is a clustering of abnormalities that confers an increased risk of developing not only cardiovascular disease (CVD) but also type 2 diabetes mellitus [1]. It has reached to epidemic proportions worldwide [2]. About $20-30 \%$ of adult population worldwide is suffering from this syndrome [3]. Millions of people in developing countries are facing a double health burden that represents an unsettling modern-day paradox i.e. the impact of poverty-related diseases (associated with infections and nutrition) is being exacerbated by the increasing load of chronic non communicable diseases [4].

MS was firstly defined by World Health Organization (WHO) in 1998 [5], after that many international agencies and organizations purposed various definitions to screen it. Out of these the most widely used definitions are by the National Cholesterol Education Program Expert Panel on Detection, Evaluation, and Treatment

\footnotetext{
* Correspondence: chetna9709@yahoo.co.in
${ }^{1}$ Department of Community Medicine, Government Medical College

* Correspondence: chetna9709@yahoo.co.in
'Department of Community Medicine, Government Medical College Chandigarh, India
}

(c) 2010 Mangat et al; licensee BioMed Central Ltd. This is an Open Access article distributed under the terms of the Creative Commons Attribution License (http://creativecommons.org/licenses/by/2.0), which permits unrestricted use, distribution, and reproduction in any medium, provided the original work is properly cited. in 2001 [6] and the International Diabetes Federation (IDF) proposed in 2005 [7]. But all the groups agreed on the core components of MS that consist of obesity, insulin resistance, dyslipidemia and hypertension, however the specific factors and their cutoff values used in the various definitions were different to identify MS.

According to a recent systematic review of studies published between 1998 and 2005, has shown the strong association between the MS and the risk of CVD [8]. MS also increases incidence of coronary heart diseases and diabetes [9]. Apart from its association with cardiovascular disorder and diabetes mellitus, it is a common soil for numerous other clinical disorders too and so it has became a matter of great debate.

The prevalence of MS is rapidly increasing in developing countries due to changing lifestyle. Chandigarh is a highly urbanized city ( $90 \%$ of population resides in urban areas) and there seems to be no literature available regarding MS in Chandigarh except a small school based study in adolescents [10]. The present community based study was conducted to screen individuals from 
rural, urban and slum populations of Chandigarh along with its associated factors.

\section{Objectives}

1. To determine the prevalence of Metabolic Syndrome in adults aged 18 years and above in Chandigarh, India. 2. To determine socio-demographic and some other factors associated with MS. 3. To determine the agreement between IDF and ATP-III.

\section{Study design}

A community based cross-sectional study was conducted in urban and rural and slum populations of Union Territory, Chandigarh, located in northern India.

\section{Methods}

Stratified multistage random sampling was used for selection of study subjects. The whole population was divided in to rural and urban strata. At the first stage, sample of 2 rural and 6 urban wards were selected at random as primary stage units. Within each primary stage unit, a sample of one sector in urban area and one village from rural area were selected at random as secondary stage units. Within selected second stage units a sample of households were surveyed as third stage units. Within selected households, individuals satisfying inclusion criteria were selected to achieve the pre-determined optimum sample size.

In India the prevalence of MS as available in different studies, ranges from $20 \%-40 \%$. On the basis of pilot study conducted prevalence of $40 \%$ was found, by keeping $95 \%$ confidence coefficient and allowing $10 \%$ permissible error the optimum sample size was found to be 576 . Total 606 subjects agreed for biochemical investigations and one blood sample was hemolysed during transportation hence 605 respondents were screened for MS.

Individuals aged 18 yrs and above, irrespective of disease status (diabetics, hypertensives and dyslipidemic patients were not excluded) were screened for MS, only pregnant females were excluded from screening. For the sake of feasibility in terms of time, cost and other considerations, IDF and ATP-III criteria were used for screening of MS [6,7]. Key points regarding these two criteria are given in table 1 and table 2 .

Participants were interviewed and examined clinically to get required information. Semi structured schedule was used to gather information regarding background characteristics and lifestyle related information. Socioeconomic status was classified using Modified Kuppuswamy Socio-economic status scale [11]. Physical activity was assessed on the basis of occupation and accordingly it was classified in to sedentary, moderate and heavy type [12]. Anthropometric measurements which include waist circumference, weight, and height were done using
Table 1 ATP-III definition of Metabolic Syndrome

\begin{tabular}{ll}
\hline \multicolumn{2}{l}{ Three or more of the following five risk factors: } \\
\hline Risk factor & Defining level \\
\hline Central obesity & Waist circumference \\
- Men & $>102 \mathrm{~cm}(>40 \mathrm{in})$ \\
- Women & $>88 \mathrm{~cm}(>35 \mathrm{in})$ \\
\hline Triglycerides & $\geq 150 \mathrm{mg} / \mathrm{dL}(1.7 \mathrm{mmol} / \mathrm{L})$ \\
\hline HDL cholesterol & $<40 \mathrm{mg} / \mathrm{dL}(1.03 \mathrm{mmol} / \mathrm{L})$ \\
- Men & $<50 \mathrm{mg} / \mathrm{dL}(1.29 \mathrm{mmol} / \mathrm{L})$ \\
- Women & $\geq 130 / \geq 85 \mathrm{~mm} \mathrm{Hg}$ \\
\hline Blood pressure & $\geq 110 \mathrm{mg} / \mathrm{dL}(6.1 \mathrm{mmol} / \mathrm{L})$ \\
\hline Fasting glucose
\end{tabular}

standard methods. Body Mass Index (BMI) was classified according to WHO classification. Blood pressure measurements were done as per JNC VII guidelines [13]. Required biochemical parameters: triglycerides (TG), high density lipids (HDL) and fasting blood sugar (FBS) levels were estimated in participants who remained fasting for 12 hours prior to sample collection. The blood sample was collected using standard blood sample collection procedure. After labeling blood sample vials, they were transported to biochemistry laboratory of Government Medical College and Hospital Chandigarh for further processing.

Statistical tests like Chi square test, Normal tests of proportions, Student's t-test, and Analysis of variance (ANOVA) were applied. Kappa coefficient was used for studying agreement between IDF and ATP-III criteria [14]. SPSS-12 software was used for data analysis.

Ethical guidelines of Helsinki (1996) were followed. Informed consent was taken from all the participants and any risks involved during investigation were cared of medically to the possible extent. Information regarding MS was also provided to the participants prior to survey. Participants were imparted knowledge regarding prevention of MS after conducting the interview.

\section{Results}

Out of 605 participants, 290 (47.9\% were males) and 315 (52.1\% were females). During sampling, proportional allocation was given to urban, rural and slum populations, as $481(79.5 \%), 63(10.4 \%)$, and 61 (10.1\%) participants were from respective populations. Participants from all of the socioeconomic classes and religions were included in this study. Table 3 and Table 4 show the prevalence of MS and its association with various sociodemographic factors by IDF and ATP-III criteria respectively. According to IDF, 287 (47.4\%) respondents were having MS and it was more in females $171(54.3 \%)$ as compared to males 116 (40\%). Prevalence estimates were less using ATP-III, as 233 (38.5\%) individuals had 


\section{Table 2 IDF definition of Metabolic Syndrome}

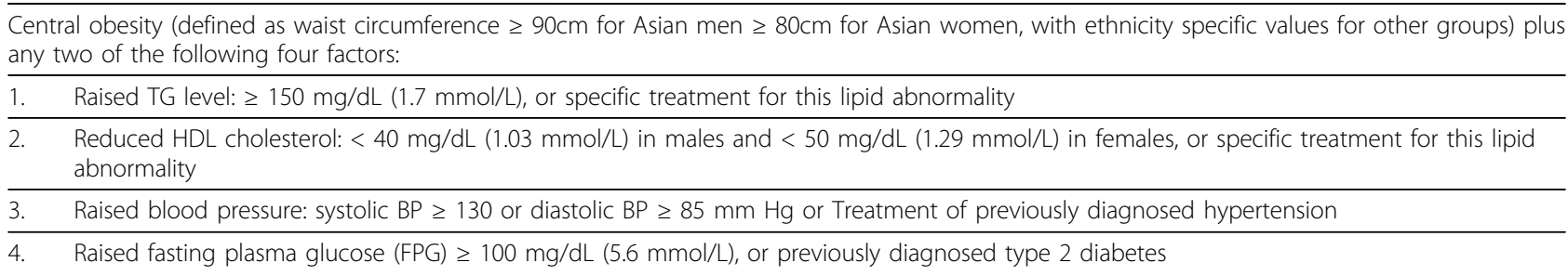

MS. However, similar trends in favor of the female gender were seen, as 141 (44.8\%) females compared to 116 (31.7\%) males were having MS.

Mean age of study population was $44.99 \pm 14.74 \mathrm{yrs}$, but it was more in respondents with MS with both criteria. It was $49.46 \pm 13.24$ yrs in respondents with MS according to IDF $(\mathrm{p}<.001)$ and $50.55 \pm 13.24 \mathrm{yrs}$ in respondents with MS according to ATP-III ( $<<.001)$. Increasing trends of prevalence with age were seen with both IDF and ATP-III.

MS was more prevalent in urban area, by using both criteria. About 249 (51.8\%) urban residents were having MS (IDF) as compared to $26(41.3 \%)$ rural residents and $12(19.7 \%)$ slum dwellers $(\mathrm{p}<.001)$. Similarly, prevalence of MS (ATP-III) was also more in urban residents $198(41.2 \%)$ as compared to $22(34.9 \%)$ rural residents and $13(21.3 \%)$ slum dwellers $(\mathrm{p}=.009)$.

MS was more prevalent among respondents belonging to upper socioeconomic as compared to lower classes and there association was statistically significant ( $\mathrm{p}=$ .006 and $\mathrm{p}=.021$ respectively for IDF and ATP-III). MS was more prevalent among respondents belonging to Sikh religion. Prevalence was $58.2 \%$ in Sikhs followed by Hindus (42.2\%), Christians (40\%) and Muslims (35\%) by IDF and it was statistically significant $(\mathrm{p}=.002)$. By ATP-III prevalence among Sikhs was $45.2 \%$, Hindus (35.2\%), Christians (40\%), and Muslims (30\%) but this association was statistically insignificant $(\mathrm{p}=.100)$.

It has been found that type of occupation, is significantly associated with MS (IDF) as 259 (51.4\%) respondents involved in sedentary habitat were having MS as compared to $28(30.1 \%)$ respondents of moderate type of work and none of the respondents involved in heavy work were having MS ( $\mathrm{p}<.001)$. Parallel results were found by using ATP-III too. As 211 (41.9\%) participants involved in sedentary habitat MS as compared 22 (23.7\%) involved in moderate type of physical activity and none of the respondents involved in heavy activity had MS ( $<$.001).

Highly significant association of BMI with MS has been found by using both IDF and ATP-III criteria. None of the respondents from underweight group and only 51 (19.2\%) individuals with normal BMI had MS (IDF). Prevalence of MS (IDF) increased from $67.5 \%$ in respondents from preo-obese class to $100 \%$ in respondents of obese class III. This association was highly significant $(\mathrm{p}<.001)$. Same trends were found by using ATP-III also ( $<$.001). Mean BMI of study population was low i.e. $25 \pm 4.13$ yrs as compared to the respondents with MS (IDF) $27.89 \pm 3.02$ years and respondents with MS (ATP-III) $27.95 \pm 4.15$ years.

The agreement between ATP-III and IDF criteria was found by using kappa coefficient of agreement and it was 0.636 and it is highly significant $(\mathrm{P}<.001)$.

\section{Discussion}

\section{Prevalence world wide IDF vs. NCEP ATP-III}

In the present study prevalence of MS was estimated by using both IDF and ATP-III criteria and overall prevalence rates were found as $47.5 \%$ and $38.5 \%$ respectively. Higher prevalence (by using IDF) can be explained by the lower cut-off points adopted by this new definition. In IDF, central obesity is the major criterion, its cut off is ethnic specific and is lower for Indians than used by original ATP-III. Another difference among two definitions is lower cutoff for FBS by IDF, which is $>100$ as compared to > 110 in ATP-III.

The higher prevalence by new IDF definitions is comparable with other reports. $\mathrm{Hu}$ et al conducted a study in Finland and found that prevalence was $39.1 \%$ by ATP-III and $45.3 \%$ by IDF definition [15]. Similarly study done by Can et al concluded that prevalence was $42 \%$ by IDF and $38 \%$ by ATP-III in Turkish adults [16]. Similar results were also found by Harzallah et al [17] in Arab population ( $45.5 \%$ by IDF vs. $24.3 \%$ by ATP-III).

\section{Prevalence in India}

In India very few studies had been done to find prevalence of MS and most of the studies which are available in literature had used ATP-III. But Deepa et al [18] compared the prevalence of MS in south Indian population by various definitions and found that by IDF $25.8 \%$ individuals > 20 yrs were having MS as compared to $18.3 \%$ by ATP-III. Another study from Bangalore by Kanjlal et al [19] concluded that prevalence of MS (by ATP-III) was $40.3 \%$ as compared to $34.9 \%$ by IDF definition. But no comparable study from north India is present in literature which has compared these two definitions. Gupta et al estimated the prevalence in Bhatia 
Table 3 Metabolic Syndrome (IDF) with various socio-demographic factors

\begin{tabular}{|c|c|c|c|c|}
\hline \multirow[t]{2}{*}{ Socio demographic factor } & \multicolumn{2}{|c|}{$\begin{array}{l}\text { Metabolic Syndrome } \\
\text { (IDF) }\end{array}$} & \multirow[t]{2}{*}{$\begin{array}{l}\text { Total } \\
\text { No. (\% age) }\end{array}$} & \multirow[t]{2}{*}{$P$ value } \\
\hline & $\begin{array}{l}\text { Yes } \\
\text { No. (\% age) }\end{array}$ & $\begin{array}{l}\text { No } \\
\text { No.(\% age) }\end{array}$ & & \\
\hline \multicolumn{5}{|l|}{ Gender } \\
\hline Male & $\begin{array}{l}116(40.4) \\
(40.0)^{*}\end{array}$ & $\begin{array}{l}174(54.7) \\
(60.0)^{*}\end{array}$ & $\begin{array}{l}290(47.9) \\
(100.0)^{*}\end{array}$ & $\mathrm{p}<.001$ \\
\hline Female & $\begin{array}{l}171(59.6) \\
(54.3)\end{array}$ & $\begin{array}{l}144(45.3) \\
(45.7)\end{array}$ & $\begin{array}{l}315(52.1) \\
(100.0)\end{array}$ & \\
\hline \multicolumn{5}{|l|}{$\overline{\text { Age }}$} \\
\hline $18-25$ yrs & $\begin{array}{l}5(1.7) \\
(9.4)\end{array}$ & $\begin{array}{l}48(15.1) \\
(90.6)\end{array}$ & $\begin{array}{l}53(8.8) \\
(100.0)\end{array}$ & $\mathrm{p}<.001$ \\
\hline $26-35$ yrs & $\begin{array}{l}35(12.2) \\
(30.7)\end{array}$ & $\begin{array}{l}79(24.8) \\
(60.3)\end{array}$ & $\begin{array}{l}114(18.8) \\
(100.0)\end{array}$ & \\
\hline $36-49 \mathrm{yrs}$ & $\begin{array}{l}114(39.7) \\
(50.7)\end{array}$ & $\begin{array}{l}111(34.9) \\
(49.3)\end{array}$ & $\begin{array}{l}225(37.2) \\
(100.0)\end{array}$ & \\
\hline $50-59$ yrs & $\begin{array}{l}56(19.5) \\
(61.5)\end{array}$ & $\begin{array}{l}35(11.0) \\
(38.5)\end{array}$ & $\begin{array}{l}91(15.0) \\
(100.0)\end{array}$ & \\
\hline $60-65$ yrs & $\begin{array}{l}42(14.6) \\
(63.6)\end{array}$ & $\begin{array}{l}24(7.5) \\
(36.4)\end{array}$ & $\begin{array}{l}66(10.9) \\
(100.0)\end{array}$ & \\
\hline$>65$ yrs & $\begin{array}{l}35(12.2) \\
(62.5)\end{array}$ & $\begin{array}{l}21(6.6) \\
(37.5)\end{array}$ & $\begin{array}{l}56(9.3) \\
(100.0)\end{array}$ & \\
\hline Mean \pm SD & $49.46 \pm 13.24$ & $44.96 \pm 14.88$ & $44.99 \pm 14.74$ & \\
\hline \multicolumn{5}{|l|}{ Background } \\
\hline Urban & $\begin{array}{l}249(86.8) \\
(51.8)\end{array}$ & $\begin{array}{l}232(73.0) \\
(48.2)\end{array}$ & $\begin{array}{l}481(79.5) \\
(100.0)\end{array}$ & $\mathrm{p}<.001$ \\
\hline Rural & $\begin{array}{l}26(9.1) \\
(41.3)\end{array}$ & $\begin{array}{l}37(11.6) \\
(58.7)\end{array}$ & $\begin{array}{l}63(10.4) \\
(100.0)\end{array}$ & \\
\hline Slum & $\begin{array}{l}12(4.2) \\
(19.7)\end{array}$ & $\begin{array}{l}49(15.4) \\
(80.3)\end{array}$ & $\begin{array}{l}61(10.1) \\
(100.0)\end{array}$ & \\
\hline \multicolumn{5}{|l|}{ Socioeconomic status } \\
\hline Upper-upper & $\begin{array}{l}9(3.1) \\
(64.3)\end{array}$ & $\begin{array}{l}5(1.6) \\
(35.7)\end{array}$ & $\begin{array}{l}14(8.8) \\
(100.0)\end{array}$ & $p=.006$ \\
\hline Upper-middle & $\begin{array}{l}190(66.2) \\
(51.6)\end{array}$ & $\begin{array}{l}178(56.0) \\
(48.4)\end{array}$ & $\begin{array}{l}368(60.8) \\
(100.0)\end{array}$ & \\
\hline Upper-lower & $\begin{array}{l}55(19.2) \\
(44.4)\end{array}$ & $\begin{array}{l}69(21.7) \\
(55.6)\end{array}$ & $\begin{array}{l}124(20.5) \\
(100.0)\end{array}$ & \\
\hline Lower & $\begin{array}{l}33(11.5) \\
(33.3)\end{array}$ & $\begin{array}{l}66(17.7) \\
(66.7)\end{array}$ & $\begin{array}{l}99(16.4) \\
(100.0)\end{array}$ & \\
\hline \multicolumn{5}{|l|}{ Religion } \\
\hline Hindu & $\begin{array}{l}157(54.7) \\
(42.2)\end{array}$ & $\begin{array}{l}215(67.6) \\
(57.8)\end{array}$ & $\begin{array}{l}372(61.5) \\
(100.0)\end{array}$ & $p=.002$ \\
\hline$\overline{\text { Sikh }}$ & $\begin{array}{l}121(42.2) \\
(58.2)\end{array}$ & $\begin{array}{l}87(27.4) \\
(41.8)\end{array}$ & $\begin{array}{l}208(34.4) \\
(100.0)\end{array}$ & \\
\hline Muslim & $\begin{array}{l}7(2.4) \\
(35.0)\end{array}$ & $\begin{array}{l}13(4.1) \\
(65.0)\end{array}$ & $\begin{array}{l}20(3.3) \\
(100.0)\end{array}$ & \\
\hline Christian & $\begin{array}{l}2(0.7) \\
(40.0)\end{array}$ & $\begin{array}{l}3(0.9) \\
(60.0)\end{array}$ & $\begin{array}{l}5(0.8) \\
(100.0)\end{array}$ & \\
\hline \multicolumn{5}{|l|}{ Physical activity } \\
\hline Sedentary & $\begin{array}{l}259(90.2) \\
(51.4)\end{array}$ & $\begin{array}{l}245(77.0) \\
(48.6)\end{array}$ & $\begin{array}{l}504(83.3) \\
(100.0)\end{array}$ & $p<.001$ \\
\hline Moderate & $\begin{array}{l}28(9.8) \\
(30.1)\end{array}$ & $\begin{array}{l}65(20.4) \\
(69.9)\end{array}$ & $\begin{array}{l}93(15.4) \\
(100.0)\end{array}$ & \\
\hline Heavy & $\begin{array}{l}0(0.0) \\
(0.0)\end{array}$ & $\begin{array}{l}8(2.5) \\
(100.0)\end{array}$ & $\begin{array}{l}8(1.3) \\
(100.0)\end{array}$ & \\
\hline
\end{tabular}


Table 3: Metabolic Syndrome (IDF) with various socio-demographic factors (Continued)

\begin{tabular}{llll}
\hline BMI & & & \\
\hline Underweight & $0(0.0)$ & $18(5.7)$ & $18(3.0)$ \\
$(0.0)$ & $(100.0)$ & $266(44.0)$ \\
\hline Normal & $51(17.8)$ & $215(67.6)$ & $(100.0)$ \\
\hline Pre-obese & $(19.2)$ & $(80.8)$ & $240(39.7)$ \\
& $162(56.4)$ & $78(24.5)$ & $(100.0)$ \\
\hline Obese Class I & $(67.5)$ & $(32.5)$ & $69(11.4)$ \\
& $63(22.0)$ & $6(1.9)$ & $(100.0)$ \\
\hline Obese Class II & $(91.3)$ & $(8.7)$ & $10(1.7)$ \\
& $9(3.1)$ & $1(0.3)$ & $(100.0)$ \\
\hline Obese Class III & $(90.0)$ & $(10.0)$ & $2(0.3)$ \\
& $2(0.7)$ & $0(0.0)$ & $(100.0)$ \\
\hline Mean \pm SD & $(100.0)$ & $(0.0)$ & $25.40 \pm 4.13$ \\
\hline Total & $27.89 \pm$ & $23.16 \pm 3.17$ & $605(100.0)$ \\
& $287(100.0)$ & $318(100.0)$ & $(100.0)$ \\
\hline
\end{tabular}

*Percentage in second row (in bold letters) represents percentage within that group in Table 3 and Table 4

community in Rajasthan by ATP-III definition and it was $36.2 \%$ in males and $47.8 \%$ in females [20].

Reddy et al [21] conducted a multicentric study in subjects 20-69 yrs of age belonging to industrial employees or their family members. They used ATP-III and higher prevalence was found in Bangalore i.e. $38.8 \%$ comparable to current study (38.5\%), Trivandrum $37.9 \%$, Hyderabad 33.0\%, Lucknow 29.0\% but lower prevalence was found in Nagpur and Dibrugarh High prevalence of MS in present study could be attributed to modern life style adopted by residents of a highly urbanized population of Chandigarh, an Union Territory of India,

\section{MS with age and gender}

In this study prevalence of MS (IDF) was highest i.e. $63.6 \%$ in age group of $60-65$ years followed by age group of $>65$ years i.e. $62.5 \%$ and lowest among age group of $18-25$ years i.e. $9.4 \%$. Prevalence was more among females than males by both IDF $(\mathrm{M}=40.0 \%, \mathrm{~F}=$ $54.3 \%)$ and ATP-III $(\mathrm{M}=31.7 \%, \mathrm{~F}=44.8 \%)$. An overall increasing trend was reported in prevalence rates with increasing age by both definitions. This finding was in concordance with the study conducted by Kanjlal et al in Bangalore [19], who reported maximum prevalence in age group of 50-59 years and Reddy et al [21] who reported maximum prevalence among age group $>60$ years in multi-centric industrial population of India. Similar trends were also reported by a study done by Taylor et al [22] in African-American population, who studied the prevalence in age group 21-94 years and maximum prevalence was seen in 65-74 year age group and lowest was seen among 21-34 year age group. Prevalence of MS was $44.8 \%$ in females and $33.4 \%$ among males. Another study done by Hildrum et al [23] in Norwegian population also found the similar trends in prevalence according to age and gender.

\section{Urban Rural Prevalence comparison}

Prevalence of MS was highest among urban residents (51.8\%, 41.2\%) followed by rural population (41.3\%, 34.9\%) and prevalence in slum residents was lowest $(19.7 \%$, 21.3\%) by both IDF and ATP-III definitions. But interestingly prevalence of MS by ATP-III was more among slum residents as compared to IDF definition. It could be due to less prevalence of central obesity among slum residents because of less sedentary lifestyle and central obesity is a major criterion in IDF as compared to ATP-III. Mahadik et al [24] also compared the prevalence among urban and rural populations of India and prevalence was more among urban as compared to rural residents (35.2\% vs. 20.6\%). Ramirez-Vargas et al [25] also found that prevalence of MS was more in urban as compared to rural populations (45.4\% vs. 27.6\%) in Oaxaca, Mexico. Another study by Sarkar et al [26] also revealed the effect of urbanization by comparing the prevalence of MS among two tribes of sub-Himalayan region of India.

\section{MS with Religion}

Present study also showed the significant correlation between MS and religion. Sikh community had highest prevalence of MS (58.2\%, 45.2\%) followed Hindus (42.2\%, $35.2 \%$ ) by both IDF and ATP-III definitions but maximum number of respondents were from Hindu religion (61.5\%). This difference occurred probably because of difference in the dietary habits of different communities. No comparable study could be found in the literature. 
Table 4 Metabolic Syndrome (ATP-III) with various socio-demographic factors

\begin{tabular}{|c|c|c|c|c|}
\hline \multirow[t]{2}{*}{ Socio demographic factor } & \multicolumn{2}{|c|}{$\begin{array}{c}\text { Metabolic Syndrome } \\
\text { (ATP-III) }\end{array}$} & \multirow[t]{2}{*}{$\begin{array}{l}\text { Total } \\
\text { No. (\% age) }\end{array}$} & \multirow[t]{2}{*}{$p$ value } \\
\hline & $\begin{array}{l}\text { Yes } \\
\text { No. (\% age) }\end{array}$ & $\begin{array}{l}\text { No } \\
\text { No. (\% age) }\end{array}$ & & \\
\hline \multicolumn{5}{|l|}{ Gender } \\
\hline Male & $\begin{array}{l}116(39.5) \\
(31.7)\end{array}$ & $\begin{array}{l}198(53.2) \\
(68.3)\end{array}$ & $\begin{array}{l}290(47.9) \\
(100.0)\end{array}$ & $p<.001$ \\
\hline Female & $\begin{array}{l}141(44.8) \\
(44.8)\end{array}$ & $\begin{array}{l}174(46.8) \\
(55.2)\end{array}$ & $\begin{array}{l}315(52.1) \\
(100.0)\end{array}$ & \\
\hline \multicolumn{5}{|l|}{ Age } \\
\hline $18-25$ yrs & $\begin{array}{l}4(1.7) \\
(7.5)\end{array}$ & $\begin{array}{l}49(13.2) \\
(92.5)\end{array}$ & $\begin{array}{l}53(8.8) \\
(100.0)\end{array}$ & $\mathrm{p}<.001$ \\
\hline $26-35$ yrs & $\begin{array}{l}25(10.7) \\
(21.9)\end{array}$ & $\begin{array}{l}89(23.9) \\
(78.1)\end{array}$ & $\begin{array}{l}114(18.8) \\
(100.0)\end{array}$ & \\
\hline $36-49 \mathrm{yrs}$ & $\begin{array}{l}87(37.3) \\
(38.7)\end{array}$ & $\begin{array}{l}138(37.1) \\
(61.3)\end{array}$ & $\begin{array}{l}225(37.2) \\
(100.0)\end{array}$ & \\
\hline $50-59$ yrs & $\begin{array}{l}48(20.6) \\
(52.7)\end{array}$ & $\begin{array}{l}43(11.6) \\
(47.3)\end{array}$ & $\begin{array}{l}91(15.0) \\
(100.0)\end{array}$ & \\
\hline $60-65$ yrs & $\begin{array}{l}38(16.3) \\
(57.6)\end{array}$ & $\begin{array}{l}28(7.5) \\
(42.4)\end{array}$ & $\begin{array}{l}66(10.9) \\
(100.0)\end{array}$ & \\
\hline$>65$ yrs & $\begin{array}{l}31(13.3) \\
(55.4)\end{array}$ & $\begin{array}{l}25(6.7) \\
(44.6)\end{array}$ & $\begin{array}{l}56(9.3) \\
(100.0)\end{array}$ & \\
\hline Mean \pm SD & $50.55 \pm 13.24$ & $41.51 \pm 14.58$ & $44.99 \pm 14.74$ & \\
\hline \multicolumn{5}{|l|}{ Background } \\
\hline Urban & $\begin{array}{l}198(85.0) \\
(41.2)\end{array}$ & $\begin{array}{l}283(76.1) \\
(58.8)\end{array}$ & $\begin{array}{l}481(79.5) \\
(100.0)\end{array}$ & $p=.009$ \\
\hline Rural & $\begin{array}{l}22(9.4) \\
(34.9)\end{array}$ & $\begin{array}{l}41(11.0) \\
(65.1)\end{array}$ & $\begin{array}{l}63(10.4) \\
(100.0)\end{array}$ & \\
\hline Slum & $\begin{array}{l}13(5.6) \\
(21.3)\end{array}$ & $\begin{array}{l}48(12.9) \\
(78.7)\end{array}$ & $\begin{array}{l}61(10.1) \\
(100.0)\end{array}$ & \\
\hline \multicolumn{5}{|l|}{ Socioeconomic status } \\
\hline Upper-upper & $\begin{array}{l}9(3.9) \\
(64.3)\end{array}$ & $\begin{array}{l}5(1.3) \\
(35.7)\end{array}$ & $\begin{array}{l}14(8.8) \\
(100.0)\end{array}$ & $p=.021$ \\
\hline Upper-middle & $\begin{array}{l}153(65.7) \\
(41.6)\end{array}$ & $\begin{array}{l}215(57.8) \\
(58.4)\end{array}$ & $\begin{array}{l}368(60.8) \\
(100.0)\end{array}$ & \\
\hline Upper-lower & $\begin{array}{l}38(16.3) \\
(30.6)\end{array}$ & $\begin{array}{l}86(23.1) \\
(55.6)\end{array}$ & $\begin{array}{l}124(20.5) \\
(100.0)\end{array}$ & \\
\hline Lower & $\begin{array}{l}33(14.2) \\
(33.3)\end{array}$ & $\begin{array}{l}66(17.7) \\
(66.7)\end{array}$ & $\begin{array}{l}99(16.4) \\
(100.0)\end{array}$ & \\
\hline \multicolumn{5}{|l|}{ Religion } \\
\hline Hindu & $\begin{array}{l}131(56.2) \\
(35.2)\end{array}$ & $\begin{array}{l}241(64.8) \\
(64.8)\end{array}$ & $\begin{array}{l}372(61.5) \\
(100.0)\end{array}$ & $p=.100$ \\
\hline$\overline{\text { Sikh }}$ & $\begin{array}{l}94(40.3) \\
(45.2)\end{array}$ & $\begin{array}{l}114(30.6) \\
(54.8)\end{array}$ & $\begin{array}{l}208(34.4) \\
(100.0)\end{array}$ & \\
\hline Muslim & $\begin{array}{l}6(2.6) \\
(30.0)\end{array}$ & $\begin{array}{l}14(3.8) \\
(70.0)\end{array}$ & $\begin{array}{l}20(3.3) \\
(100.0)\end{array}$ & \\
\hline Christian & $\begin{array}{l}2(0.9) \\
(40.0)\end{array}$ & $\begin{array}{l}3(0.8) \\
(60.0)\end{array}$ & $\begin{array}{l}5(0.8) \\
(100.0)\end{array}$ & \\
\hline \multicolumn{5}{|l|}{ Physical activity } \\
\hline Sedentary & $\begin{array}{l}211(90.6) \\
(41.9)\end{array}$ & $\begin{array}{l}293(78.8) \\
(58.1)\end{array}$ & $\begin{array}{l}504(83.3) \\
(100.0)\end{array}$ & $\mathrm{P}<.001$ \\
\hline Moderate & $\begin{array}{l}22(9.4) \\
(23.7)\end{array}$ & $\begin{array}{l}71(19.1) \\
(76.3)\end{array}$ & $\begin{array}{l}93(15.4) \\
(100.0)\end{array}$ & \\
\hline Heavy & $\begin{array}{l}0(0.0) \\
(0.0)\end{array}$ & $\begin{array}{l}8(2.2) \\
(100.0)\end{array}$ & $\begin{array}{l}8(1.3) \\
(100.0)\end{array}$ & \\
\hline
\end{tabular}


Table 4: Metabolic Syndrome (ATP-III) with various socio-demographic factors (Continued)

\begin{tabular}{|c|c|c|c|c|}
\hline \multicolumn{4}{|l|}{ BMI } & \multirow[b]{2}{*}{$\mathrm{P}<.001$} \\
\hline Underweight & $\begin{array}{l}1(0.4) \\
(5.6)\end{array}$ & $\begin{array}{l}17(4.6) \\
(94.4)\end{array}$ & $\begin{array}{l}18(3.0) \\
(100.0)\end{array}$ & \\
\hline Normal & $\begin{array}{l}51(21.9) \\
(19.2)\end{array}$ & $\begin{array}{l}215(57.8 \%) \\
(80.8)\end{array}$ & $\begin{array}{l}266(44.0) \\
(100.0)\end{array}$ & \\
\hline Pre-obese & $\begin{array}{l}110(47.2) \\
(45.8)\end{array}$ & $\begin{array}{l}130(34.9) \\
(54.2)\end{array}$ & $\begin{array}{l}240(39.7) \\
(100.0)\end{array}$ & \\
\hline Obese Class I & $\begin{array}{l}60(25.8) \\
(87.0)\end{array}$ & $\begin{array}{l}9(2.4) \\
(13.0)\end{array}$ & $\begin{array}{l}69(11.4) \\
(100.0)\end{array}$ & \\
\hline Obese Class II & $\begin{array}{l}9(3.9) \\
(90.0) \\
\end{array}$ & $\begin{array}{l}1(0.3) \\
(10.0)\end{array}$ & $\begin{array}{l}10(1.7) \\
(100.0)\end{array}$ & \\
\hline Obese Class III & $\begin{array}{l}2(0.9) \\
(100.0) \\
\end{array}$ & $\begin{array}{l}0(0.0) \\
(0.0) \\
\end{array}$ & $\begin{array}{l}2(0.3) \\
(100.0) \\
\end{array}$ & \\
\hline Mean \pm SD & $27.95 \pm 4.15$ & $23.81 \pm 3.23$ & $25.40 \pm 4.13$ & \\
\hline Total & $\begin{array}{l}233(100.0) \\
(38.5)\end{array}$ & $\begin{array}{l}372(100.0) \\
(61.5)\end{array}$ & $\begin{array}{l}605(100.0) \\
(100.0\end{array}$ & \\
\hline
\end{tabular}

\section{MS with Socioeconomic Status}

This study had shown the significant association of MS with socioeconomic status of respondents. MS was found to be more prevalent among subjects belonging to upper socioeconomic status as compared to lower classes. This finding was in concordance to the study done by Mohan et al [27] in South India where in significant difference in prevalence of MS was found according to socioeconomic status. MS was higher in middle compared to low income groups But in studies from developed countries and have shown the contrast results as Dallongeville et al [28] found that household income and education had an inverse relation with MS in France.

\section{Agreement of IDF and NCEP ATP-III}

Significant agreement between ATP-III and IDF criteria for detecting MS indicates that we can use either of these two criteria for detecting MS. Optimum decisions may depend upon epidemiological situations. Choi et al [29], reported the agreement between ATP-III and the IDF as $(\kappa=0.54)$ in the Korean population.

\section{Conclusions}

This study concludes that Metabolic Syndrome is highly prevalent in the urbanized community. Its contributory complications demand, screening of individuals in the community at the earliest, so that lifestyle modifications strategies may be adopted at an early age. Significant agreement between ATP-III and IDF criteria suggests possible use of IDF which restricts the blood investigations only if of central obesity is present as it is a major criterion. IDF may be a more feasible, practical and cost effective approach in the community set-up. This preliminary study in the region with IDF definition sets the background for future prospective studies, regarding causation, prevention and management of this syndrome.

\section{Limitations}

In this study only demographic factors are presented, risk factor analysis should have been done by using prospective study.

\section{Acknowledgements}

CM really wants to thank Department of Biochemistry, Government Medical College Chandigarh and her family for their cooperation.

\section{Author details}

${ }^{1}$ Department of Community Medicine, Government Medical College Chandigarh, India. ${ }^{2}$ Department of Biochemistry, Government Medical College Chandigarh, India. ${ }^{3}$ Department of Medicine, Government Medical College Chandigarh, India. ${ }^{4}$ Goondiwindi Medical Centre, Goondiwindi, QLD, Australia.

\section{Authors' contributions}

$\mathrm{CM}$ was responsible for the conception of the research question, collection, analysis and interpretation of data; and writing of the manuscript. NKG, MKS, NA and RS were responsible for conception of the research question, study design and coordination of research project. DKW was responsible for statistical analysis, interpretation of data and provided critical feedback to the manuscript for important intellectual content. JK participated in systematic biochemical analysis of the project. GS assisted in data collection, drafting of the manuscript and revised it critically for important intellectual content. All authors read and approved the final manuscript.

\section{Competing interests}

The authors declare that they have no competing interests.

Received: 31 July 2009 Accepted: 23 March 2010

Published: 23 March 2010

\section{References}

1. Wilson WF, Agostino R, Parise H, Sullivan L, Meigs J: Metabolic Syndrome as a precursor of Cardiovascular Disease and Type 2 Diabetes Mellitus. Circulation 2005, 112:3066-72.

2. Gotto AM Jr, Blackburn GL, Dailey GE III, Garber AJ, Grundy SM, Sobel BE, et al: The metabolic syndrome: A call to action. Coron Artery Dis 2006, 17:77-80. 
3. Grundy SM: Metabolic syndrome pandemic. Arterioscler Thromb Vasc Biol 2008, 28(4):629-36.

4. Mohan V, Deepa M: The metabolic syndrome in developing countries. Diabetes Voice 2006, 51:15-17.

5. Alberti KG, Zimmet PZ: Definition, diagnosis and classification of diabetes Mellitus and its complications. Diagnosis and classification of diabetes mellitus provisional report of a WHO consultation. Diabet Med 1998, 15:539-553.

6. Executive Panel on Detection Evaluation and Treatment of High Blood Cholesterol in Adults: Executive Summary of the Third Report of the National Cholesterol Education Program (NCEP) Expert Panel on Detection, Evaluation, and Treatment of High Blood Cholesterol in Adults (Adult Treatment Panel III). JAMA 2001, 285:2486-2497.

7. International Diabetes Federation: The IDF consensus worldwide definition of the metabolic syndrome. 2001 [http://www.idf.org/webdata/docs/ Metabolic_syndrome_definition.pdf], Accessed April 3, 2006.

8. Earl S, Ford ES: Risks for all-cause mortality, cardiovascular disease, and diabetes associated with the metabolic syndrome: A summary of the evidence. Diabetes Care 2005, 28:1769-78.

9. Klein B, Klein R, Lee K: Components of the Metabolic Syndrome and risk of Cardiovascular Disease and Diabetes in Beaver Dam. Diabetes Care 2002, 25:1790-4.

10. Singh R, Bhansali A, Sialy R, Aggarwal A: Prevalence of metabolic syndrome in adolescents from a north Indian population. Diabetic Medicine 2007, 24(2):195-99.

11. Kuppuswamy B: Manual of Socio-Economic Scale (Urbans). Ind J Pediatrics 2003, 70.

12. Gopalan C, Sastri Rama BV, Balasubramanian, Rao Narasinga BS, Deosthale YG, Pant KC: Nutritive Value Ind Foods. 2003.

13. The Seventh Report of the Joint National committee on Prevention, Detection, Evaluation, and Treatment of High Blood Pressure. .

14. Nonparametric statistics for the behavioral sciences. McGraw-hill international editions statistical series, 2.

15. Hu G, Lindström J, Jousilahti P, Peltonen M, Sjöberg L, Kaaja R, Sundvall J, Tuomilehto J: The increasing prevalence of metabolic syndrome among Finnish men and women over a decade. J Clin Endocrinol Metab 2008, 93(3):832-6.

16. Can AS, Bersot TP: Analysis of agreement among definitions of metabolic Syndrome in nondiabetic Turkish adults: a methodologicalstudy. BMC Public Health 2007, 7:353.

17. Harzallah F, Alberti H, Ben Khalifa F: The metabolic syndrome in an Arab Population: a first look at the new International Diabetes Federation criteria. Diabet Med 2006, 23(4):441-4.

18. M Deepa, S Farooq, M Datta, R Deepa, V Mohan: Prevalence of metabolic syndrome using WHO, ATP-III and IDF definitions in Asian Indians: the Chennai Urban Rural Epidemiology Study (CURES-34). Diabetes/ Metabolism Research and Reviews 2006, 23(2):127-134

19. Kanjilal S, Shanker J, Rao VS, Khadrinarasimhaih N, Mukherjee M, lyengar SS, et al: Prevalence and component analysis of metabolic syndrome: An Indian atherosclerosis research study perspective. Vascular Health and Risk Management 2008, 4(1):189-197.

20. Gupta R, Sarna M, Thanvi J, Rastogi P, Kaul V, Gupta VP: High prevalence of multiple coronary risk factors in Punjabi Bhatia community: Jaipur Heart Watch-3. Indian Heart J 2004, 56(6):646-52

21. Reddy KS, Prabhkaran D, Chaturvedi, et al: Methods for establishing a surveillance system for cardiovascular diseases in Indian industrial populations-Bulletin of WHO/June. 2004, 84(6):461-69.

22. Taylor H, Liu J, Wilson G, Golden SH, Crook E, Brunson CD, et al: Distinct component profiles and high risk among African Americanswith metabolic syndrome: the Jackson Heart Study. Diabetes Care 2008, 31(6):1248-53.

23. Hildrum B, Mykletun A, Hole T, Midthjell K, Dahl AA: Age-specific prevalence of the metabolic syndrome defined by the International Diabetes Federation and the National Cholesterol Education Program: the Norwegian HUNT 2 study. BMC Public Health 2007, 7:220.

24. Mahadik SR, Deo SS, Mehtalia SD: Increased prevalence of metabolic syndrome in non-obese Asian Indian-an urban-ruralcomparison. Metab Syndr Relat Disord 2007, 5(2):142-52.

25. Ramírez-Vargas E, Arnaud-Viñas Mdel R, Delisle H: Prevalence of the metabolic syndrome and associated lifestyles in adult males from Oaxaca, Mexico. Salud Publica Mex 2007, 49(2):94-102.
26. Sarkar S, Das M, Mukhopadhyay, Chakarbarti C, Majumdar P: High prevalence of metabolic syndrome \& its correlates in two tribal populations of India \& the impact of urbanization. Indian JMed Res123 2006, 679-86.

27. Mohan V, Shanthirani S, Deepa R, Premalatha G, Sastry NG, Saroja R: Intraurban difference in prevalence of the metabolic syndrome in southern India-the Chennai Urban Ppulation Study (CUPS No. 4). Diabetes Med 2001, 18(4):280-7.

28. Dallongeville J, Cottel $D$, Ferrières J, Arveiler $D$, Bingham A, Ruidavets JB, et al: Household Income Is Associated With the Risk of Metabolic Syndrome in a Sex-Specific Manner. Diabetes Care 2005, 28: 409-415.

29. Choi K, Kim S, Kim Y, Choi D, Baik S, Lee J: Prevalence and cardiovascular disease risk of the metabolic syndrome using National Cholesterol Education Program and International Diabetes Federation definitions in the Korean population. Metabolism 2007, 56(4):552-8.

doi:10.1186/1758-5996-2-19

Cite this article as: Mangat et al: Metabolic Syndrome: a challenging health Issue in highly urbanized Union Territory of north India. Diabetology \& Metabolic Syndrome 2010 2:19.

\section{Submit your next manuscript to BioMed Central and take full advantage of:}

- Convenient online submission

- Thorough peer review

- No space constraints or color figure charges

- Immediate publication on acceptance

- Inclusion in PubMed, CAS, Scopus and Google Scholar

- Research which is freely available for redistribution
C Biomed Central 